\title{
Thickness dependence of amplified spontaneous emission in low-absorbing organic waveguides
}

\author{
Eva M. Calzado, ${ }^{1,2}$ Manuel G. Ramírez,, ${ }^{1,3}$ Pedro G. Boj, ${ }^{1,4}$ and María A. Díaz García ${ }^{1,3, *}$ \\ ${ }^{1}$ Instituto Universitario de Materiales de Alicante (IUMA) and Unidad Asociada UA-CSIC, \\ Universidad de Alicante, 03080-Alicante, Spain \\ ${ }^{2}$ Departamento de Física, Ingeniería de Sistemas y Teoría de la Señal; Universidad de Alicante, 03080-Alicante, Spain \\ ${ }^{3}$ Departamento de Física Aplicada, Universidad de Alicante, 03080-Alicante, Spain \\ ${ }^{4}$ Departamento de Óptica, Farmacología y Anatomía; Universidad de Alicante, 03080-Alicante, Spain \\ *Corresponding author: maria.diaz@ua.es
}

Received 23 February 2012; revised 30 March 2012; accepted 30 March 2012;

posted 2 April 2012 (Doc. ID 163592); published 24 May 2012

\begin{abstract}
The effect of varying film thickness $(h)$ on the amplified spontaneous emission (ASE) properties of $0.5 \mathrm{wt} . \%$ perylenediimide-doped polystyrene waveguides is reported. The threshold dependence on $h$, not previously investigated in detail, is analyzed in terms of the film absorption and photoluminescence, the confinement of the fundamental waveguide mode $\left(\mathrm{TE}_{0}\right)$, and the presence of high-order modes. For $h<$ $400 \mathrm{~nm}$ and down to $150 \mathrm{~nm}$, the ASE wavelength blueshifts, while the linewidth and threshold increase. The detrimental ASE operation in very thin films is due to the low absorption as well as to the poor confinement of the $\mathrm{TE}_{0}$ mode. (C) 2012 Optical Society of America
\end{abstract}

OCIS codes: $\quad 140.0140,140.2050,140.3580,160.0160,160.3380,160.4890$.

\section{Introduction}

Numerous studies have been devoted to the search of materials with optimal properties to be applied in the development of organic solid-state lasers (OSLs) [1-4]. This interest is based on the various advantages offered by organic materials: easy processability in the form of thin films, chemical versatility, wavelength tunability, and low cost. Since the discovery of stimulated emission in semiconducting polymer films [ [ -7$]$, most efforts have focused on decreasing the laser thresholds in view of using the electrical pump. Although diode lasers have not been demonstrated yet, optical excitation with cheap inorganic diode lasers [3] and even with light-emitting diodes [8] has been reported. Therefore, these lowcost an $\bar{d}$ compact optically pumped lasers are by themselves useful for applications $[3,4]$.

$1559-128 \mathrm{X} / 12 / 163287-07 \$ 15.00 / 0$

(C) 2012 Optical Society of America
The easiest way to identify the presence of stimulated emission in a certain organic material and to evaluate its potential for laser applications consists of characterizing its amplified spontaneous emission (ASE) when deposited as a thin film in a waveguide configuration $[2,9]$. Typically, active films are photopumped with a pulsed optical source, aiming to identify a collapse of the width of their photoluminescence (PL) spectra at a certain pump intensity. This spectral narrowing is normally accompanied by a large enhancement of the output intensity and accounts for the presence of gain due to stimulated emission. In addition, this technique is the most convenient for comparing the performance of different materials since it allows separating variations due to the material from those due to the resonant cavity.

The ASE properties of films of a wide variety of organic materials have been reported in the literature [2-4]. Many of them have also been used to build laser devices, including resonators of various kinds, i.e., microcavities, distributed feedback (DFB) structures, 
microrings, etc. Among all these materials, our group has focused in the last years in polystyrene (PS) doped with perylenediimide derivatives (PDIs) [10-13], mainly due to their excellent thermal and photostability properties, as well as their high PL quantum efficiencies. In addition, these materials are particularly interesting in the field of data communications based on polymer optical fibers because they emit at wavelengths around $570 \mathrm{~nm}$, inside the second low-loss transmission window in poly(methylmethacrylate). The optical properties (i.e., absorption, PL emission, and ASE) for various PDI derivatives with different chemical structure were first investigated in solution and dispersed in PS in the form of thin films. It was concluded that PDIs substituted at the imide nitrogen positions had the best performance [10]. Then, with one of these derivatives, the effect on the ASE properties of changing the PDI concentration in the film was investigated. The lowest thresholds and longest photostability half-lives were obtained with doping PDI percentages between 0.5 and $1 \mathrm{wt} . \%$ [11]. Recently, these materials were used to prepare very efficient and photostable second-order, onedimensional DFB lasers, with gratings on the substrate [12] and on the active film [13]. Despite all this work, the dependence of the ASE properties of PDIdoped PS films on film thickness $(h)$ has not been studied.

In fact, in the general context of OSLs, there have been just a few investigations about the film thickness dependence of the ASE properties of organic waveguides [14-17]. These reports have focused on tuning the ASE emission wavelength $\left(\lambda_{\mathrm{ASE}}\right)$ by changing $h$. It was shown that when $h$ was close to the cutoff thickness ( $\left.h_{\text {cut-off }}\right)$ for the propagation of one mode, $\lambda_{\mathrm{ASE}}$ shifted to shorter wavelengths. Tuning ranges up to around $30 \mathrm{~nm}$ were achieved with various semiconducting polymers [14-16], while shorter values $(13 \mathrm{~nm})$ were obtained with PS doped with 15 wt.\% of the organic molecule TPD [17]. However, ASE threshold data were only provided in two of these publications $[16,17]$. It was found that threshold increased up to around one order of magnitude when decreasing film thickness. In [17], this increase was interpreted in terms of the confinement of the waveguide modes. But other parameters that might play a role, such as the absorption and PL emission of the film or the presence of high order modes, were not considered.

Despite the limited amount of information regarding the film thickness dependence of the ASE properties on waveguides, there have been numerous reports investigating the influence of this parameter in the context of organic DFB lasers [13,18-22]. This is because varying $h$ constitutes the simplest way to tune the emission wavelength $\left(\lambda_{\mathrm{DFB}}\right)$ of the devices, tunability being one of the most attracting properties of organic materials as laser active media. However, there is an important difference between ASE and DFB emission when analyzing this effect. In the case of waveguides without resonator, for a wide range of thickness (far from $h_{\text {cut-off }}$ of the $\mathrm{TE}_{0}$ mode), $\lambda_{\mathrm{ASE}}$ appears at approximately the same spectral position (very close to the maximum of the gain spectrum). On the other hand, in DFB lasers, $\lambda_{\mathrm{DFB}}$ is determined by the grating period and by the effective index $\left(n_{\text {eff }}\right)$ of the film. This latter parameter depends on the thickness and refractive index of the active film, the refractive index of the substrate and the cladding layer, and the wavelength of the light propagating along the waveguide. So whenever $h$ increases (for a given period), $\lambda_{\mathrm{DFB}}$ also increases. The available tuning range is limited by the gain spectral width and at lower wavelengths by the overlap with the absorption spectrum. Consequently, in the literature in which the influence of $h$ on the DFB emission threshold has been analyzed, results have been explained in terms of the proximity of the emission wavelength to the maximum of the gain spectrum $[13,18-22]$. In some cases, it was suggested that some other parameters, such as the confinement of the waveguide mode $[13,18]$ or the presence of competing higher order modes [19], could play a role. However, a detailed analysis at this respect was not done.

In addition, there is another parameter that might considerably influence the thresholds of both ASE and DFB emission that has not generally been considered before. That is the fact that optical absorption, and consequently PL intensity, varies significantly when $h$ is changed. In this respect, the behavior will be different depending on the type of active media. Two extreme cases would be (1) lowabsorbing materials, such as inert polymer films doped with a small percentage of active molecule and (2) highly absorbing materials, such as neat active molecular or polymer films. In the first case, the intensity of the excitation beam over the sample would keep practically constant with depth, so absorption and PL intensity would increase linearly with $h$. On the other hand, for highly absorbing materials, the intensity of the pump beam would decay rapidly with depth, so after a certain $h$ value, the absorbance, and consequently the generated PL intensity, would no longer increase. PS films doped with 0.5 wt.\% of PDI belong to the first category, while semiconducting polymers, which have been the most widely studied systems in the last few years, fall in the second category.

Within this context, we aimed to investigate in detail the influence on the ASE properties (i.e. emission wavelength, linewidth, and threshold) of changing film thickness in PDI-doped PS waveguides. The effect on the threshold, not previously investigated in detail, has been analyzed in terms of film optical absorption and PL emission, the confinement of the fundamental waveguide mode, and the presence of high-order modes.

\section{Experimental Methods}

The samples were prepared by the spin-coating technique. Toluene solutions containing PS (Sigma Aldrich, $\mathrm{M}_{\mathrm{w}}=35000 \mathrm{~g} / \mathrm{mol}$ ) as an inert polymer, 
doped with 0.5 wt.\% of the PDI derivative N, N'di-(1-hexylheptyl) perylene-3,4:9,10-tetracarboxylic diimide, PDI-C6 (LambdaChem, $\mathrm{M}_{\mathrm{w}}=755 \mathrm{~g} / \mathrm{mol}$ ), were prepared and cast over transparent $\mathrm{SiO}_{2}$ substrates. The chemical structure of PDI-C6 is shown in the inset of Fig. 1. The percentage of PS with respect to the solvent was adjusted in order to obtain $h$ values between 90 and $1200 \mathrm{~nm}$. $h$ was measured by means of an interferometer coupled to an optical microscope. Films of good optical quality (transparency and homogeneity) were found to be stable for many months when stored at room temperature in air and in the dark.

These films constitute asymmetric planar waveguides since their refractive index $\left(n_{f}\right)$ is larger than that of the $\mathrm{SiO}_{2}$ substrate $\left(n_{s}\right)$ and the cover (air, $n_{c}$ ). Since the concentration of PDI-C6 is very small, $n_{f}$ was calculated from the dispersion relation of undoped PS films, given by $n_{f}=A+\left(B / \lambda^{2}\right)+\left(C / \lambda^{4}\right)$, with $A=1.5691, B=0.0052, C=0.0007$, and with $\lambda$ expressed in micrometers [23]. Concerning $n_{s}$, it was obtained from $n_{s}^{2}=1+\left[A_{1} \lambda^{2} /\left(\lambda^{2}-C_{1}^{2}\right)\right]+$ $\left[A_{2} \lambda^{2} /\left(\lambda^{2}-C_{2}^{2}\right)\right]$, where $A_{1}=1.0245, A_{2}=0.0422$, $C_{1}=0.1355, C_{2}=0.0289$, and with $\lambda$ expressed in micrometers. This latter dispersion relation was experimentally determined by fitting data measured at various wavelengths, provided by a mercury lamp, with an Abbe refractometer. Particularly, at $\lambda=579 \mathrm{~nm}, n_{f}=1.59$ and $n_{s}=1.46$. The waveguiding properties of the films were both measured and modeled. Experimental $n_{\text {eff }}$ values associated with the transverse electric (TE) and transverse magnetic (TM) modes propagating in the structure were determined by the $m$-line technique [24], using as a light source a mercury lamp emitting at $\lambda=578 \mathrm{~nm}$. Theoretical $n_{\text {eff }}$ values were calculated by solving the propagation wave equation, following well-known methods $[17,25]$.

Absorption measurements were carried out in a Jasco V-650 spectrophotometer. The absorption coefficient for a film of thickness $h$, at a given wavelength $\alpha_{\lambda}$, was calculated according to $\alpha_{\lambda}=2.3 A_{\lambda} / h$, where $A_{\lambda}$ was the absorbance. This expression was obtained from $A_{\lambda}=-\log \left(I / I_{0}\right)$, in which $I$ and $I_{0}$ were the transmitted and incident beams respectively and, considering the Lambert-Beer's law, given by $\left(I / I_{0}\right)=\exp (-\alpha h)$. Standard PL spectra were obtained in a Jasco FP-6500/6600 fluorimeter, collecting the transmitted beam at a $45^{\circ}$ angle. The excitation wavelength was $491 \mathrm{~nm}$, instead of the $532 \mathrm{~nm}$ beam used in the ASE experiments, in order to reduce the overlap between absorption and emission.

The ASE properties of the films were explored by optical excitation with a frequency-doubled Nd:YAG (YAG-yttrium aluminum garnet) laser (10 ns, $10 \mathrm{~Hz})$ operating at $532 \mathrm{~nm}$, which lays close to the maximum absorbance of PDI-C6. A scheme of the experimental setup can be found in [26]. The energy of the pulses was varied using neutral density filters. The pump laser beam was expanded and collimated, and only the central part was selected in order to ensure uniform intensity. A cylindrical lens and an adjustable slit were then used to shape the beam into a stripe of approximately $530 \mu \mathrm{m}$ by $3.5 \mathrm{~mm}$. The stripe was placed right up to the edge of the film, from where PL emission was collected with an Ocean Optics USB2000-UV-VIS fiber spectrometer with 600 grating lines and a resolution in determining the emission linewidth of $1.3 \mathrm{~nm}$. The precision in measuring the emission wavelength is around half of this value.

\section{Results and Discussion}

\section{A. Absorption, PL, and ASE Spectra}

ASE was observed in 0.5 wt.\% PDI-C6-doped PS films for a range of film thickness between 150 and $1200 \mathrm{~nm}$. As an illustration, Fig. 1 shows the ASE spectrum obtained for a PS film with $h=1070 \mathrm{~nm}$. The absorption and PL spectra of the film have also been included in the figure. ASE takes place at a wavelength close to the peak of the $0-1$ PL transition. These results are comparable to those previously reported for a $1 \mu \mathrm{m}$ thick PS film doped with $0.75 \mathrm{wt} . \%$ of a similar derivative, N, N'-di(10-nonadecyl)perylene3, 4:9, 10-tetracarboxylic diimide, denoted as PDI-N [11]. The chemical structure of PDI-C6 differs from that of PDI-N only in the length of the chains attached to the imide $\mathrm{N}$ positions.

Figure 2 displays the spectral linewidth, defined as the full width at half of its maximum (FWHM) as a function of pump intensity for the same film whose spectra are shown in Fig. 1. This type of plot was used to determine the ASE threshold $\left(I_{\text {th-ASE }}\right)$ as the excitation intensity at which the FWHM decays to half of its maximum value. The existence of gain results not only in a spectral narrowing at the threshold, but also in a considerable increase of the output intensity, as also shown in Fig. $\underline{2}$.

\section{B. Waveguide Characterization and Modeling}

Before discussing the effect of changing $h$ on the various ASE parameters, we present the results obtained

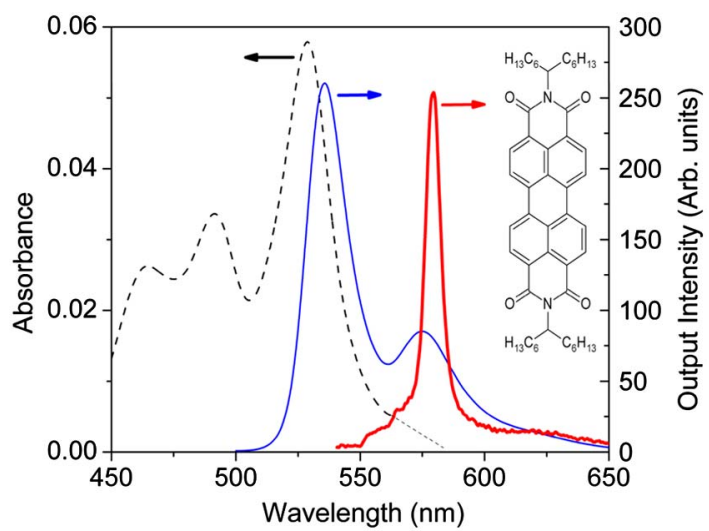

Fig. 1. (Color online) Absorbance (dashed line in left axis), PL and ASE (thin and thick full lines, respectively, in right axis) spectra of a PDI-C6-doped PS film with $h=1070 \mathrm{~nm}$. Inset, chemical structure of PDI-C6. 


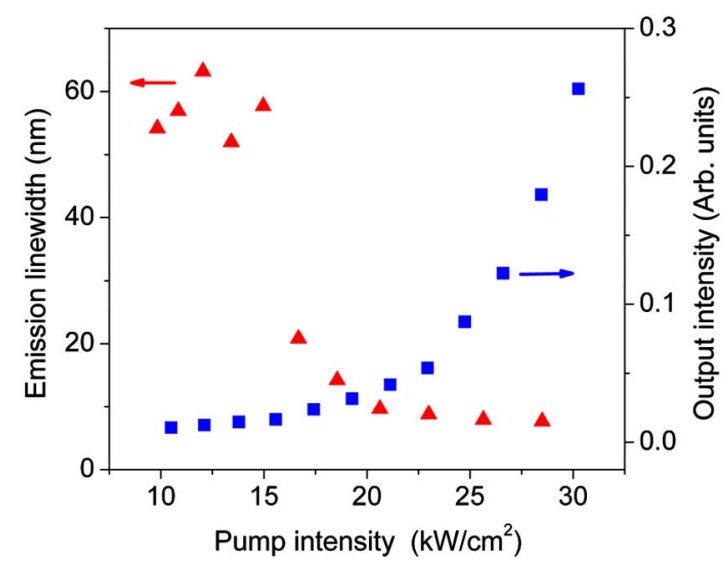

Fig. 2. (Color online) Emission linewidth (triangles in left axis) and output intensity at $579 \mathrm{~nm}$ (squares in right axis) as a function of pump intensity for a PDI-C6-doped PS film with $h=1070 \mathrm{~nm}$.

in relation to the characterization and modeling of the waveguide properties of the films since they will be needed for that purpose.

Effective indices $\left(n_{\text {eff }}\right)$ for the TE and TM modes, calculated with the propagation wave equation at $579 \mathrm{~nm}[17,25]$, are represented in Fig. 3 as a function of $h$. From these plots, some useful information can be obtained, such as the number of modes propagating in the waveguide for a given $h$ and the minimum $h$ needed for a certain mode to propagate in the waveguide, denoted as cut-off thickness $\left(h_{\text {cut-off }}\right)$. The one related with the fundamental mode, $h_{\text {cut-off }}\left(\mathrm{TE}_{0}\right)=$ $149 \mathrm{~nm}$, plays an important role when trying to tune $\lambda_{\mathrm{ASE}}$, as discussed below. For higher order modes, the calculated $h_{\text {cut-off }}$ values are 600 and $1050 \mathrm{~nm}$ for the $\mathrm{TE}_{1}$ and $\mathrm{TE}_{2}$ modes, respectively.

Experimental $n_{\text {eff }}$ values for various films are also represented in Fig. $\underline{3}$. As observed, calculations and experiments are in good agreement.

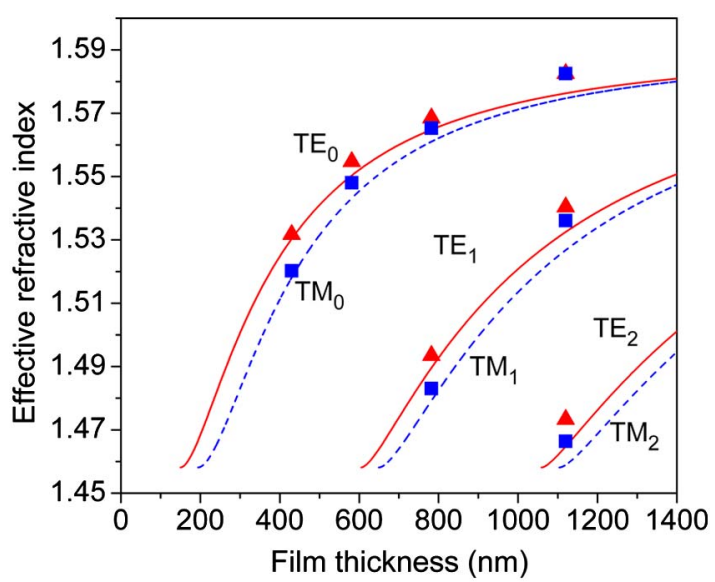

Fig. 3. (Color online) Effective refractive index $\left(n_{\text {eff }}\right)$ for transverse electric (TE) and transverse magnetic (TM) modes as a function of film thickness for PDI-C6-doped PS films deposited over $\mathrm{SiO}_{2}$ substrates. Lines are calculations with the propagation wave equation at $\lambda=579 \mathrm{~nm}$ (full and dashed lines for TE and TM modes, respectively); symbols are experimental $n_{\text {eff }}$ values determined by the $m$-line technique at $\lambda=578 \mathrm{~nm}$ (triangles and squares for TE and TM modes, respectively).
C. Thickness Dependence of the ASE Wavelength and Linewidth

The influence on the ASE wavelength $\left(\lambda_{\mathrm{ASE}}\right)$ of changing $h$ is depicted in Fig. 4 . A tuning range of around $4 \mathrm{~nm}$ (i.e., $\lambda_{\mathrm{ASE}}$ varying between 575.5 and $579.5 \mathrm{~nm}$ ) was achieved. Two regimes were observed: (1) For $h>400 \mathrm{~nm}, \lambda_{\mathrm{ASE}}$ appears at a wavelength around $579.5 \mathrm{~nm}$, and (2) for $h<400 \mathrm{~nm}$ and down to $150 \mathrm{~nm}, \lambda_{\mathrm{ASE}}$ decreases rapidly, reaching a minimum $\lambda_{\text {ASE }}$ value of $575.5 \mathrm{~nm}$. No ASE was observed for films with $h \leq 130 \mathrm{~nm}$.

In accordance with previous reports with other materials [14-17], the rapid decrease in $\lambda_{\mathrm{ASE}}$ observed in regime (2) is attributed to the proximity of $h$ to $h_{\text {cut-off }}\left(\mathrm{TE}_{0}\right)(149 \mathrm{~nm}$ in this case). In order to satisfy the cut-off condition for a given thickness, emission is forced to emit at a lower wavelength. The tuning range obtained here is rather small $\left(\Delta \lambda_{\mathrm{ASE}} \sim 4 \mathrm{~nm}\right)$, mainly because the threshold increases drastically when using very thin films, as discussed in detail in Subsection 3.D. The limitations in these materials to tune $\lambda_{\mathrm{ASE}}$ arise from the fact that optical absorption is low due to the small doping ratios used. This is in contrast with highly absorbing films, such as semiconducting polymers, in which larger tuning ranges can be obtained [14-16].

The effect on the ASE linewidth ( $\left.\mathrm{FWHM}_{\mathrm{ASE}}\right)$ of changing $h$ is also illustrated in Fig. 4. Similar $\mathrm{FWHM}_{\mathrm{ASE}}$ values (around $6 \mathrm{~nm}$ ) were obtained for $h>300 \mathrm{~nm}$. The increase observed for the thinnest films, up to $18 \mathrm{~nm}$, coincides with the blue shift of $\lambda_{\mathrm{ASE}}$ and the corresponding increase in threshold. This type of behavior is similar to that reported for other materials [15], although in that case, changes in linewidth were observed for film thickness below the cut-off thickness.

\section{Thickness Dependence of the ASE Threshold}

Figure 5 shows the effect of changing $h$ on the ASE thresholds $\left(I_{\text {th-ASE }}\right)$, determined from plots like the one displayed in Fig. $\underline{2}$. Similar $I_{\text {th-ASE }}$ values

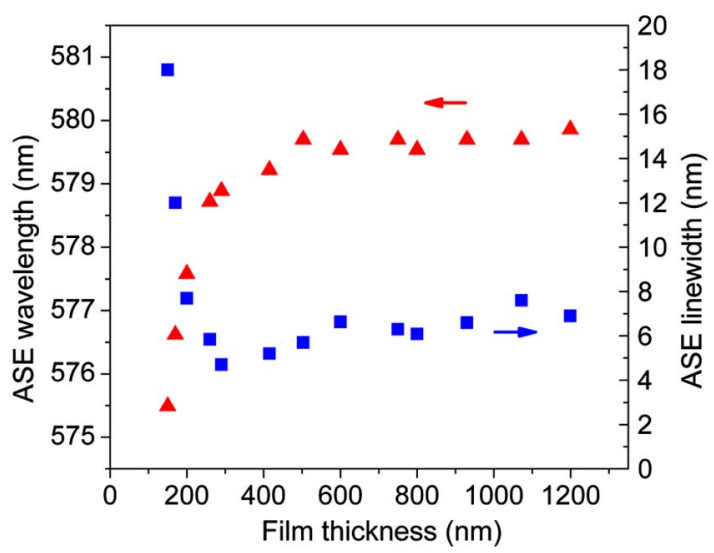

Fig. 4. (Color online) ASE wavelength and $\lambda_{\mathrm{ASE}}$ (triangles in left axis), and ASE linewidth and $\mathrm{FWHM}_{\mathrm{ASE}}$ (squares in right axis) as a function of film thickness; error is $( \pm 0.4)$ in the $\lambda_{\mathrm{ASE}}$ and $( \pm 0.8)$ in the $\mathrm{FWHM}_{\mathrm{ASE}}$. 
(between 13 and $15 \mathrm{~kW} \mathrm{~cm}^{2}$ ) were obtained for $h \geq 400 \mathrm{~nm}$, while a rapid increase was observed when $h$ decreases, reaching values as large as $1000 \mathrm{~kW} / \mathrm{cm}^{2}$ for the thinnest film with $h=170 \mathrm{~nm}$. This type of dependence is similar to that reported in the two publications where ASE threshold data as a function of $h$ are provided $[16,17]$. However, in those cases, a difference of around one order of magnitude between the highest and the lowest $I_{\text {th-ASE }}$ value was found, in contrast with the two orders of magnitude difference found in the present case. This is mainly due to the low absorption of our materials. In TPDdoped films, the increase in threshold when decreasing $h$ was explained in terms of the confinement of the fundamental mode [17]. Here, results have been interpreted by considering the influence of this parameter as well as two other aspects-the optical absorption and PL emission of the film, as well as the presence of high-order modes. As far as we know, the influence of these parameters has not been previously investigated in the context of ASE in organic waveguides.

\section{Influence of Film Optical Absorption and PL Intensity}

As shown in Fig. 6, the dependence on $h$ of both the absorbance $(A)$ at the $532 \mathrm{~nm}$ excitation wavelength and the total PL emission intensity (area under the PL spectrum) is approximately linear. This is because the PDI doping ratio used in the films is very small $(0.5 \mathrm{wt} . \%)$. So, the intensity of the excitation beam over the sample decays slowly with depth. This is illustrated in the inset of Fig. $\underline{6}$, which represents the variation of the pump beam intensity $\left[I_{p}(d)\right]$, normalized by its initial value at the air-film interface $\left[I_{p}(0)\right]$, as it travels through the film, given by $\left(I_{p}(d) / I_{p}(0)\right)=\exp (-\alpha d)$, where $d$ is the distance from the air-film interface. The absorption coefficient at $532 \mathrm{~nm}$, obtained from the absorbance fit shown in Fig. $\underline{6}$, was $\alpha_{532 \mathrm{~nm}}=1180 \mathrm{~cm}^{-1}$.

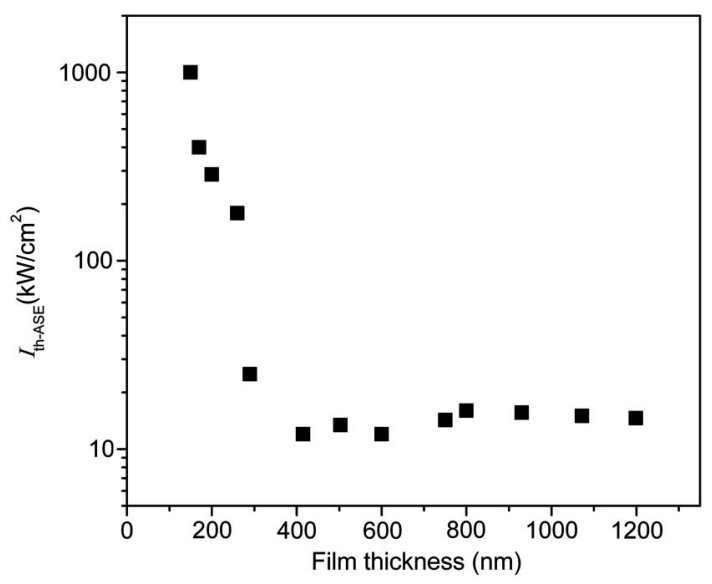

Fig. 5. ASE thresholds $\left(I_{\text {th-ASE }}\right)$, determined from plots similar to that of Fig. 2 as a function of film thickness; error in $I_{\text {th-ASE }}$ is around $10 \%$.

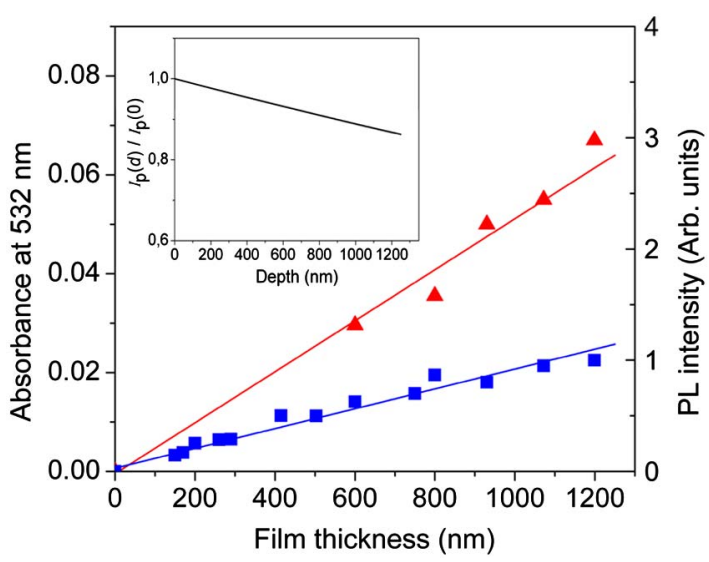

Fig. 6. (Color online) Absorbance at $532 \mathrm{~nm}$ (triangles in left axis) and total PL intensity (squares in right axis) as a function of film thickness. Straight lines are linear fits to experimental data. Inset, decay of the pump intensity with depth $(d)$ inside the film, relative to its value at the air-film interface $\left[I_{p}(d) / I_{p}(0)\right]$.

In order to quantify the influence on the threshold of the variation of the PL intensity, a parameter called "normalized threshold" $\left(I_{\text {th-ASE }}^{\text {norm }}\right)$, defined as the product of the ASE threshold by PL intensity, has been plotted in Fig. 7 for all the devices. This normalization has been performed by considering the PL emission value predicted by the linear fit (see Fig. $\underline{6}$ ) rather than using the experimental values. If the variation of PL intensity (due to different absorption) were the only relevant factor in determining the observed dependence, $I_{\text {th-ASE }}^{\text {norm }}$ should be constant with $h$. Results show that the variation of emitted PL intensity with thickness contributes significantly to the increase in threshold observed in very thin films, given that the difference between the highest and lowest $I_{\text {th-ASE }}^{\text {norm }}$ values is not as large as in the case of the non-normalized $I_{\text {th-ASE }}$ ones (150 and 1000, respectively). However, $I_{\text {th-ASE }}^{\text {norm }}$ is still larger in the thinner films. In addition, it is also observed that for very thick films, $I_{\text {th-ASE }}^{\text {norm }}$ increases slightly. Such increase could not be identified in the threshold (non-normalized) behavior.

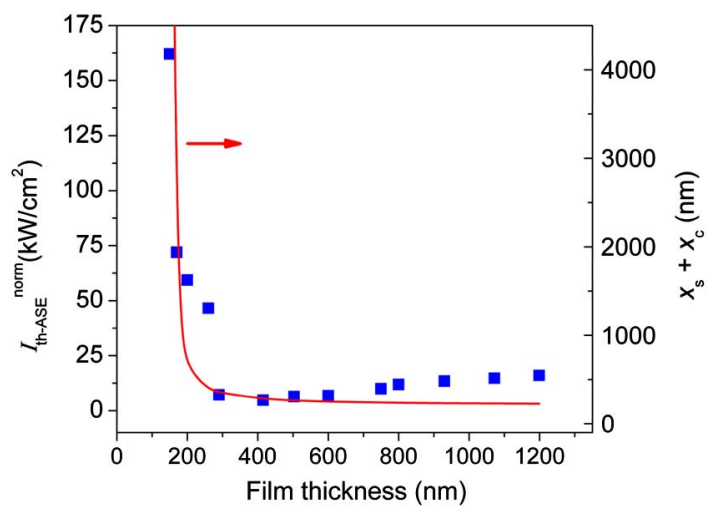

Fig. 7. (Color online) Film thickness dependence of the normalized ASE threshold ( $I_{\text {th-ASE }}^{\text {norm }}$, squares in left axis), defined as the ASE thresholds $\left(I_{\text {th-ASE }}\right)$ multiplied by the total PL intensity, and of the penetration depth in substrate $\left(x_{s}\right)$ and cover $\left(x_{c}\right)$ of the $\mathrm{TE}_{0}$ mode (full line, right axis). 
As a result of this analysis, it is concluded that the variation of emitted PL intensity is a parameter that plays an important role in the threshold variation with film thickness. Moreover, for a proper discussion of the effect of other parameters related with light propagating in the waveguide, thresholds should be normalized in order to eliminate that influence. Therefore, in what follows, discussion will be done in relation with $I_{\text {th-ASE }}^{\text {norm }}$, rather than with $I_{\text {th-ASE}}$.

\section{Influence of the Confinement of the \\ Fundamental $T E_{0}$ Waveguide Mode}

The confinement of the $\mathrm{TE}_{0}$ mode has been quantified by calculating the light penetration depth into the substrate $\left(x_{s}\right)$ and into the cover $\left(x_{c}\right.$, air) by using a simple ray model [25]. The quantity $\left(x_{s}+x_{c}\right)$, plotted in Fig. 7 as a function of $h$, is an indicator of the degree of confinement of the $\mathrm{TE}_{0}$ mode [17]- $\mathrm{a}$ large penetration depth means a low confinement of the mode and consequently high losses and a high threshold. As observed, for $h<600 \mathrm{~nm}$, the type of dependence of this parameter on $h$ is similar to that of the $I_{\mathrm{th}-\mathrm{ASE}}^{\mathrm{norm}}$, indicating its important role in its behavior, particularly for very thin films. For $h \sim 300 \mathrm{~nm}$, the penetration in the surrounding media of the only mode propagating in that case $\left(\mathrm{TE}_{0}\right)$ is as large as the thickness of the waveguide; thus, mode confinement is poor. The situation gets worse for thinner films. On the other hand, for thicker films, the penetration depth decreases, becoming small when compared to $h$. So, in this regime, its influence on the threshold is negligible. Therefore, the increase in $I_{\mathrm{th}-\mathrm{ASE}}^{\text {norm }}$ observed in Fig. 7 for $h>600 \mathrm{~nm}$ cannot be explained in terms of this parameter, since in this regime it remains practically constant. The confinement of the $\mathrm{TE}_{0}$ mode can also be analyzed by looking at the electric field profile in the waveguide structure [see Fig. 8(a)].

In summary, with respect to the influence of the confinement of the $\mathrm{TE}_{0}$ mode, the poor confinement is the reason for the increase in the normalized threshold observed in films thinner than around $300 \mathrm{~nm}$. For thicker films, this parameter does not play any important role.

\section{Influence of the Presence of High-Order} Waveguide Modes ( $T E_{1}$ and $T E_{2}$ )

The increase in $I_{\text {th-ASE }}^{\text {norm }}$ observed in Fig. 7 for $h>$ $600 \mathrm{~nm}$ seems to be due to the presence of high-order modes. This hypothesis is supported by the fact that $600 \mathrm{~nm}$ is the $h_{\text {cut-off }}$ value for the $\mathrm{TE}_{1}$ mode (see Subsection 3.B). So in films with $h>600 \mathrm{~nm}$, part of the light would travel in first-order modes, which results in an increase of $I_{\mathrm{th}-\mathrm{ASE}}^{\mathrm{norm}}$. This can be explained in terms of the waveguide losses, which are larger for these modes. This is illustrated in Fig. 8(b), which shows the electric field profile for the $\mathrm{T} \overline{\mathrm{E}}_{1}$ mode in waveguide structures of $h=800 \mathrm{~nm}$ and $h=$ $1070 \mathrm{~nm}$. For clarity, the profile of the $\mathrm{TE}_{0}$ mode has not been plotted in this figure, although for comparison purposes, it appears in Fig. 8(a) for the film

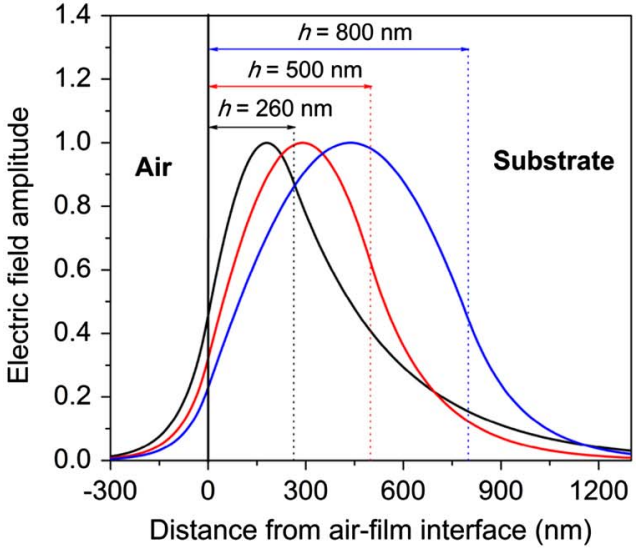

(a)

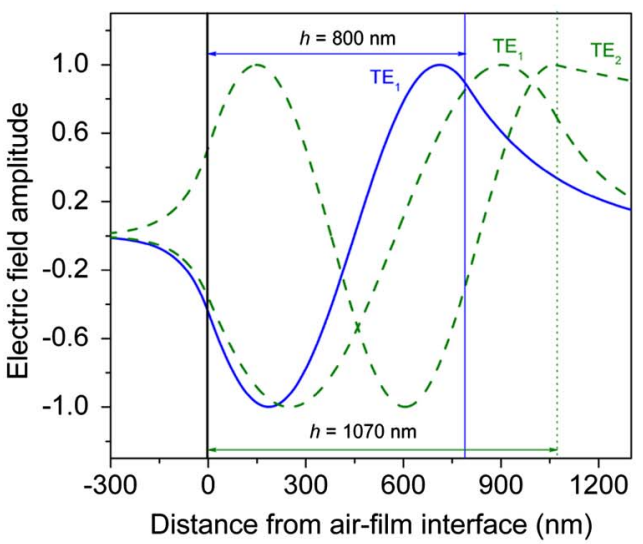

(b)

Fig. 8. (Color online) Electric field amplitude of waveguide modes as a function of the distance from the air-film interface (d): (a) $\mathrm{TE}_{0}$ mode in waveguides of $h=260,500$, and $800 \mathrm{~nm}$; (b) high-order modes in waveguides of $h=800 \mathrm{~nm}$ ( $\mathrm{TE}_{1}$, full line) and $h=$ $1070 \mathrm{~nm}\left(\mathrm{TE}_{1}\right.$ and $\mathrm{TE}_{2}$, dashed lines).

with $h=800 \mathrm{~nm}$. As observed, $\mathrm{TE}_{1}$ modes travel mainly at the interfaces, so their losses are much higher than those of the $\mathrm{TE}_{0}$ mode.

Similarly, for $h>1050 \mathrm{~nm}$, which is the $h_{\text {cut-off }}$ value for the $\mathrm{TE}_{2}$ mode, second-order modes would be able to propagate in the waveguides. However, no significant changes in the behavior of $I_{\text {th-ASE }}^{\text {norm }}$ were observed above this thickness. The electric field profile of the $\mathrm{TE}_{2}$ mode has also been plotted in Fig. 8(b) for the film with $h=1200 \mathrm{~nm}$. As observed, the losses associated with this mode were also large, particularly at the interface with the substrate.

The influence of this parameter on the ASE threshold of organic waveguides has not been previously considered. In fact, in those works that reported ASE threshold data as a function of film thickness [16-17], no significant changes in threshold were observed in thick films, although threshold normalization to eliminate the influence of different absorption and PL emission was not done. Moreover, optical absorption in those materials was higher, so one would expect saturation effects above a certain film thickness.

It can be concluded that the presence of higher order modes is detrimental in the ASE performance in 
terms of threshold. Nevertheless, the effect is not very significant and in fact cannot be appreciated in the experimental threshold values (non-normalized) since it is masked by the absorption and PL efficiency of the films that also vary with film thickness.

\section{Conclusions}

The effect of changing film thickness $(h)$, between 150 and $1200 \mathrm{~nm}$, on the ASE properties of low-absorbing organic waveguides, based on PS doped with $0.5 \mathrm{wt} . \%$ of a PDI derivative (PDI-C6), has been investigated in detail. Films with $h>400 \mathrm{~nm}$ showed ASE at $\lambda_{\mathrm{ASE}} \sim 579 \mathrm{~nm}$, with $\mathrm{FWHM}_{\mathrm{ASE}} \sim 6 \mathrm{~nm}$ and $I_{\mathrm{th}-\mathrm{ASE}} \sim$ $14 \mathrm{~kW} / \mathrm{cm}^{2}$. For $h<400 \mathrm{~nm}, \lambda_{\mathrm{ASE}}$ decreases and FWHM $_{\mathrm{ASE}}$ increases, reaching for $h=150 \mathrm{~nm}$, values of around 575 and $18 \mathrm{~nm}$, respectively. This behavior is explained by the proximity of $h$ to the cut-off thickness for the propagation of the $\mathrm{TE}_{0}$ mode. The small tuning range achieved $\left(\Delta \lambda_{\mathrm{ASE}} \sim 4 \mathrm{~nm}\right)$ is as a consequence of the low absorption of the materials, which results in a large increase in $I_{\text {th-ASE }}$, up to $1000 \mathrm{~kW} / \mathrm{cm}^{2}$, for the thinnest film.

The threshold dependence on $h$, not previously investigated in detail, has been analyzed in terms of various parameters. Results have shown that (1) film optical absorption and PL emission contributes significantly to the large increase in threshold observed in thin films and needs to be considered for a proper analysis of the effect of factors related with waveguide propagation, (2) the confinement of the $\mathrm{TE}_{0}$ mode is responsible (in combination with the low absorption) for the increase in threshold observed in thin films, and (3) the presence of high-order modes, for $h$ above the cut-off thickness for the propagation of the $\mathrm{TE}_{1}$ mode, leads to a slight increase in the threshold.

These results are useful for optimizing the laser threshold of DFB devices based on low-absorbing active materials, such as inert polymer films doped with small percentages of active molecule, in which the laser wavelength is tuned by $h$ variation.

We are thankful of the support from the Spanish Government (MEC) and the European Community (FEDER) through grants MAT2008-06648-C02 and MAT2011-28167-C02 and from the University of Alicante through grant GRE10-07. M. G. R. is supported by a CSIC fellowship within the program JAE. We also thank Dr. J. M. Villalvilla and Dr. J. A. Quintana for useful discussions and V. Navarro-Fuster and V. Esteve for technical assistance.

\section{References}

1. N. Tessler, "Lasers based on semiconducting organic materials," Adv. Mater. 11, 363-370 (1999).

2. M. D. McGehee and A. J. Heeger, "Semiconducting (conjugated) polymers as materials for solid-state lasers," Adv. Mater. 12, 1655-1668 (2000).

3. I. D. W. Samuel and G. A. Turnbull, "Organic semiconductor lasers," Chem. Rev. 107, 1272-1295 (2007).

4. J. Clark and G. Lanzani, "Organic photonics for communications," Nat. Photon. 4, 438-446 (2010).

5. F. Hide, B. Schwartz, M. A. Díaz-García, and A. J. Heeger, "Laser emission from solutions and films containing semicon- ducting polymers and titanium dioxide nanocrystals," Chem. Phys. Lett. 256, 424-430 (1996).

6. F. Hide, M. A. Díaz-García, B. Schwartz, M. Andersson, Q. Pei, and A. J. Heeger, "Semiconducting polymers: a new class of solid-state laser materials," Science 273, 1833-1836 (1996).

7. N. Tessler, G. J. Denton, and R. H. Friend, "Lasing from conjugated-polymer microcavities," Nature 382, 695-697 (1996).

8. Y. Yang, G. A. Turnbull, and I. D. W. Samuel, "Hybrid optoelectronics: a polymer laser pumped by a nitride lightemitting diode," Appl. Phys. Lett. 92, 163306 (2008).

9. M. McGehee, R. Gupta, S. Veenstra, E. M. Miller, M. A. DíazGarcía, and A. J. Heeger, "Amplified spontaneous emission from photopumped films of a conjugated polymer," Phys. Rev. B 58, 7035-7039 (1998).

10. E. M. Calzado, J. M. Villalvilla, P. G. Boj, J. A. Quintana, R. Gómez, J. L. Segura, and M. A. Díaz-García, "Effect of structural modifications in the spectral and laser properties of perylenediimide derivatives," J. Phys. Chem. C 111, 13595-13605 (2007).

11. E. M. Calzado, J. M. Villalvilla, P. G. Boj, J. A. Quintana, R. Gómez, J. L. Segura, and M. A. Díaz-García, "Amplified spontaneous emission in polymer films doped with a perylenediimide derivative," Appl. Opt. 46, 3836-3842 (2007).

12. V. Navarro-Fuster, E. M. Calzado, P. G. Boj, J. A. Quintana, J. M. Villalvilla, M. A. Díaz-García, V. Trabadelo, A. Juarros, A. Retolaza, and S. Merino, "Highly photostable organic distributed feedback laser emitting at $573 \mathrm{~nm}$," Appl. Phys. Lett. 97, 171104 (2010).

13. M. G. Ramirez, P. G. Boj, V. Navarro-Fuster, I. Vragovic, J. M. Villalvilla, I. Alonso, V. Trabadelo, S. Merino, and M. A. DíazGarcía, "Efficient organic distributed feedback lasers with imprinted active films," Opt. Express 19, 22443-22454 (2011).

14. A. K. Sheridan, G. A. Turnbull, A. N. Safonov, and I. D. W. Samuel, "Tuneability of amplified spontaneous emission through control of the waveguide-mode structure in conjugated polymer films," Phys. Rev. B 62, R11929-R11932 (2000).

15. T.-W. Lee, O. O. Park, D. H. Choi, H. N. Cho, and Y. C. Kim, "Lowthreshold blue amplified spontaneous emission in a statistical copolymer and its blend," Appl. Phys. Lett. 81, 424-426 (2002).

16. R. Xia, G. Heliotis, Y. Hou, and D. D. C. Bradley, "Fluorenebased conjugated polymer optical gain media," Org. Electron. 4, 165-177 (2003).

17. E. M. Calzado, J. M. Villalvilla, P. G. Boj, J. A. Quintana, and M. A. Díaz-García, "Tuneability of amplified spontaneous emission through control of the thickness in organicbased waveguides," J. Appl. Phys. 97, 093103 (2005).

18. G. Heliotis, R. Xia, D. D. C. Bradley, G. A. Turnbull, I. D. W. Samuel, P. Andrew, and W. L. Barnes, "Two-dimensional distributed feedback lasers using a broadband, red polyfluorene gain medium,” J. Appl. Phys. 96, 6959-6966 (2004).

19. R. Xia, G. Heliotis, P. N. Stavrinou, and D. D. C. Bradley, "Polyfluorene distributed feedback lasers operating in the greenyellow spectral region,” Appl. Phys. Lett. 87, 031104 (2005).

20. E. M. Calzado, J. M. Villalvilla, P. G. Boj, J. A. Quintana, P. A. Postigo, and M. A. Díaz-García, "Blue, surface-emitting distributed feedback lasers based on TPD-doped films," Appl. Opt. 49, 463-470 (2010).

21. S. Riechel, U. Lemmer, J. Feldmann, S. Berleb, A. G. Mückl, W. Brütting, A. Gombert, and V. Wittwer, "Very compact tunable solid-state laser utilizing a thin-film organic semiconductor," Opt. Lett. 26, 593-595 (2001).

22. S. Klinkhammer, T. Woggon, U. Geyer, C. Vannahme, S. Dehm, T. Mappes, and U. Lemmer, "A continuously tunable lowthreshold organic semiconductor distributed feedback laser fabricated by rotating shadow mask evaporation," Appl. Phys. B 97, 787-791 (2009).

23. R. H. Boundy and R. F. Raymond, Styrene: Its Polymers, Copolymers and Derivatives (Reinhold, 1952).

24. A. Boudrioua, Photonic Waveguides: Theory and Applications (Wiley, 2009).

25. C.-L. Chen, Foundations of Guided-Wave Optics (Wiley, 2006),

26. M. A. Díaz-García, E. M. Calzado, J. M. Villalvilla, P. G. Boj, J. A. Quintana, F. Giacalone, J. L. Segura, and N. Martín, "Concentration dependence of amplified spontaneous emission in two oligo-( $p$-phenylenevinylene) derivatives,” J. Appl. Phys. 97, 063522 (2005). 